\title{
WHY IS THE “TIME DELAY CONTROVERSY IN Q0957+561 NOT YET DECIDED"?
}

\author{
V.L. OKNYANSKIJ \\ Sternberg Astronomical Ins. \\ 13 Universitetskij prospekt, 119899, Moscow, Russia
}

\begin{abstract}
The time delay in Q0957+561 remains indeterminate due to differences between statistical methods, irregular data spacings, and (possibly) microlensing.
\end{abstract}

Until now, the first gravitational lens, Q0957+561AB, has been the most attractive object for time delay determinations (see Beskin \& Oknyanskij 1995). Yet in spite of intensive efforts to measure the correct value of the time delay using long-term optical and radio monitoring, we have no time delay value that would be accepted by all specialists working in the field. The published time delays for $\mathrm{Q} 0957+561$ can be divided into three sets:

1. The time delay value is about 400-430 days (Schild and Cholfin 1986, Schild 1990, Vanderriest et al. 1989, Pelt et al. 1994, 1995).

2. The time delay value is about $520-555$ days (Beskin and Oknyanskij 1992,1995, Press et al. 1992).

3. A definite time delay value cannot be found due to gaps in the data sets and possible microlensing effects (Falco et al. 1991).

Pelt et al. (1994) published a paper entitled "The Time Delay Controversy On QSO 0957+561 Is Not Yet Decided". They discussed two possible values for time delay about 410 and 540 days, but really preferred the first of them. Then, in the next paper (Pelt et al. 1995), using new extended data (Schild and Thomson 1994, below ST94), they rejected values near 540 days as a possible time delay and concluded that the correct value is about $423 \pm 6$ days. The purpose of present paper is to explain why we cannot be sure of the Pelt et al. (1995) conclusion and that the "time delay controversy is (still) not yet decided."

The following is a brief summary of our arguments: 


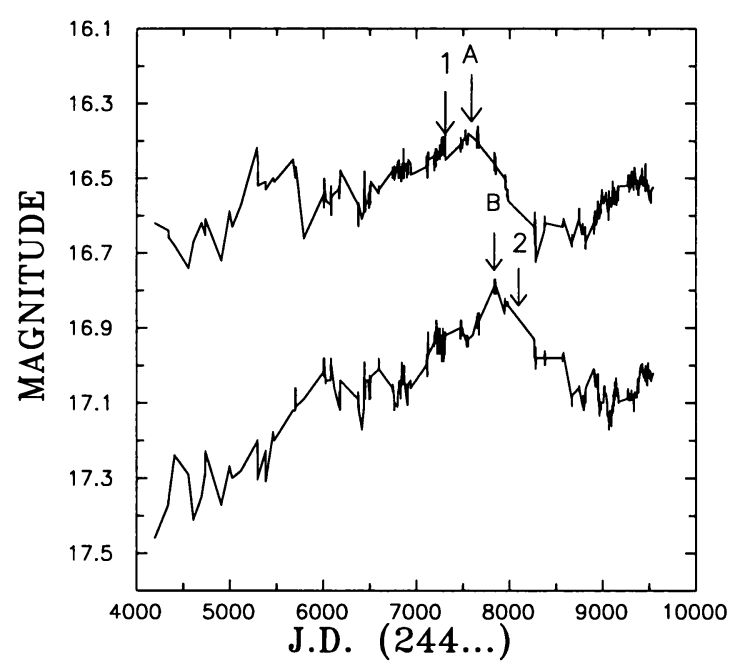

Figure 1. Optical light curves (only Schild's data) of Q0957+561 A,B. The light curve for image $B$ is shifted down by 0.5 magnitude. The 1 marks the expected location of a maximum in A light curve given the location of the maximum in the $B$ light curve and a time delay of 540 days. The 2 marks the same point for the $\mathrm{B}$ light curve.

- It was shown in Beskin and Oknyanskij $(1992,1995)$ that time delay values near 415 days may be artifacts. We should be very careful before admitting that the real time delay has about the same value expected from aliasing.

- The PDS method (Pelt et al. 1994) cannot eliminate the problem of irregular data spacing. It is simple to show that the signal/noise ratio in the PDS function depends on the time shift and must be higher for a time shift near 423 days than for one near 540 days.

- Removing trends from data sets can introduce additional noise, and give an incorrect value of the time delay.

- The spacing of weights and the density of observations in the new data set (Schild \& Thomson 1994) is very different in the first and second parts of data. The first part of the data (before J.D. 2447120) covers more time, but it is practicality negligible in the time delay analysis.

- We note (see Figure 1) that the absolute maxima and the beginning of falloffs in A and B light curves cannot be explained by either the 540-day nor the 423-day time delay. They can be explained by a time delay of about 200-300 days. We will call this interval (J.D. 24479502449170) "irregular". 


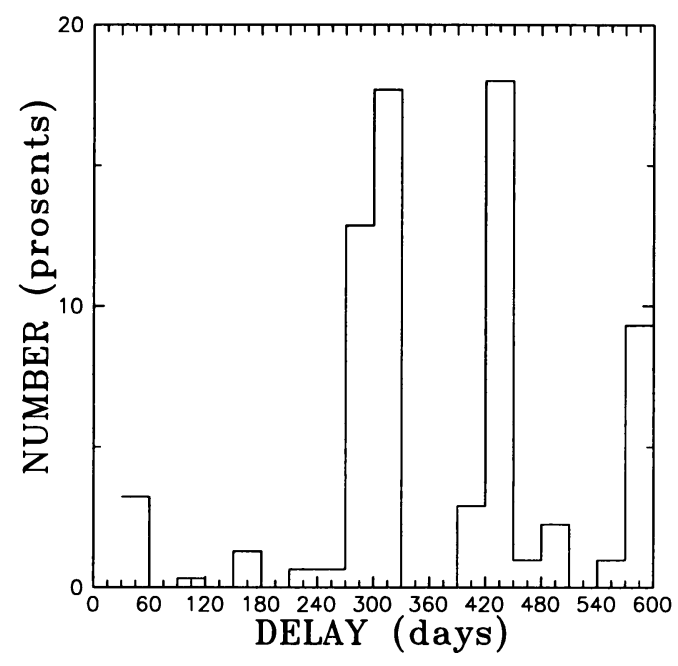

Figure 2. The distribution of time delays determined by the MCCF method (Oknyanskij 1994) for 500 Monte-Carlo simulations using the real B light curve and an artificial A light curve (see text) in the "irregular" interval.

- The autocorrelation function of the B image in the "irregular" interval has a very strong maximum of about 0.9 for a time shift near $400-440$ days. We found from Monte-Carlo simulations (using the real B light curve and simulated data consisting of white noise with some random trend in place of the real A light curve) that there is a high probability of finding a time delay of near 420 days (see Figure 2).

In conclusion, the time delay controversy for $\mathrm{Q} 0957+561$ remains undecided for two kinds of reasons:

1) subjective reasons (using different statistical methods including several methods that are not quite correct for irregularly spaced data), and

2) objective reasons (low signal/noise ratios for the time delay determinations due to periodic gaps in the data sets, possible microlensing and the small amplitude of the source variability).

Acknowledgements: The author is very thankful to R.Schild for sending photometric data before publication. This investigation has been in part financially supported by the Science Education Centre "COSMION" within the "Cosmoparticle Physics" project. 


\section{References}

Beskin, G.M., \& Oknyanskij, V.L., 1992, in Gravitational Lenses, eds. R. Kayser, T.

Schramm \& L. Nieser, (Berlin: Springer-Verlag) 67

Beskin, G.M., \& Oknyanskij, V.L. 1995, A\&A in press

Falco, E.E., Wambsganss, J., \& Schneider P., 1991, MNRAS, 251, 698

Oknyanskij, V.L., 1994, Astrophys \& Space Sci, 222, 157

Pelt, J. et al., 1994, A\&A, 256, 775

Pelt, J. et al., 1995, A\&A in press

Schild R.E., Cholfin, B., 1986, ApJ, 300, 209

Schild, R.E., 1990, AJ, 100, 1771

Schild, R.E., \& Thomson D.J., 1994, AJ in press

Vanderriest, C., Schneider, J., Herpe, G., et al., 1989, A\&A, 215, 1 